\title{
Planejamento estratégico situacional como ferramenta de promoção em saúde na gestão: revisão integrativa
}

\author{
Situational strategic planning as a health promotion tool in management: integrative review \\ La planificación estratégica situacional como herramienta de promoción de la salud en la gestión: \\ revisión integrativa
}

Recebido: 02/01/2022 | Revisado: 08/01/2022 | Aceito: 16/01/2022 | Publicado: 18/01/2022

Ellaine Valéria Araújo da Silva Lima

ORCID: https://orcid.org/0000-0001-9426-4354 Fundação Santa Casa de Misericórdia do Pará, Brasil E-mail: ellainelima11@gmail.com

Thais de Oliveira Carvalho Granado Santos

ORCID: https://orcid.org/0000-0001-9496-4561 Universidade Federal do Pará, Brasil E-mail: thaissoliveira@yahoo.com.br

Ana Gabriela Sabaa Srur de Andrade

ORCID: https://orcid.org/0000-0002-5872-6950 Fundação Santa Casa de Misericórdia do Pará, Brasil E-mail: enfgabriela@hotmail.com

Wanessa Nobre do Carmo Glória

ORCID: https://orcid.org/0000-0001-5207-4229

Fundação Santa Casa de Misericórdia do Pará, Brasil E-mail: wanessanobre19@gmail.com

José Daniel Rodrigues dos Santos

ORCID: https://orcid.org/0000-0003-1163-0502 Fundação Santa Casa de Misericórdia do Pará, Brasil E-mail: jd.enf@hotmail.com

Ilma Pastana Ferreira

ORCID: https://orcid.org/0000-0002-9152-3872 Fundação Santa Casa de Misericórdia do Pará, Brasil E-mail: pastanailma@gmail.com

Lizomar de Jesus Maues Pereira Moia

ORCID: https://orcid.org/0000-0002-6755-8725 Fundação Santa Casa de Misericórdia do Pará, Brasil E-mail: lizmoia@yahoo.com.br

Laise Cristina Pantoja Feitosa

ORCID: https://orcid.org/0000-0003-2226-6849

Fundação Santa Casa de Misericórdia do Pará, Brasil E-mail: laise.p.feitosa@gmail.com

Pilar Maria de Oliveira Moraes

ORCID: https://orcid.org/0000-0003-2817-4574 Fundação Santa Casa de Misericórdia do Pará, Brasil E-mail: pilarmoraesnutri@gmail.com

Xaene Maria Fernandes Duarte Mendonça

ORCID: https://orcid.org/0000-0002-0958-276X Fundação Santa Casa de Misericórdia do Pará, Brasil E-mail: xaenemaria@gmail.com

\begin{abstract}
Resumo
Objetivo: Avaliar como ocorre o Planejamento Estratégico Situacional na Atenção Primária à Saúde (APS) no Brasil, e de que forma ele contribui para a gestão em saúde. Método: Trata-se de uma revisão integrativa de literatura (RIL), realizada no período de novembro de 2020 a abril de 2021. Os critérios de inclusão dos estudos foram: artigos em português, inglês e espanhol, publicados nos últimos 05 anos, que apresentassem em sua discussão considerações sobre a importância do planejamento estratégico situacional na gestão dentro da APS, indexados nas bases de dados LILACS e PUBMED, utilizando-se como descritores de assunto "atenção primária à saúde" e "planejamento estratégico". Foram excluídas as publicações no formato de tese, dissertação, capítulo de livro e as que não tinham relação com o tema da pesquisa. Resultados: Foram encontradas 170 publicações. Após aplicação dos critérios de inclusão e exclusão, obteve-se 09 artigos dos quais emergiram temas quanto à importância do Planejamento Estratégico Situacional e como ele deve ser articulado em diversas áreas dentro da gestão na APS, promovendo metas
\end{abstract}


e indicadores capazes de avaliar e monitorar resultados no planejamento. Conclusão: Os resultados desta revisão encontraram diferentes formas de aplicação do Planejamento Estratégico Situacional (PES) na gestão da atenção primária a saúde com foco na promoção e prevenção, os quais mostraram que ainda existem muitos desafios a serem enfrentados, porém, possíveis de serem trabalhados através da integração ensino e serviço, da corresponsabilidade dos participantes e da aprimoração desta habilidade de gestão em profissionais que atuam nas lideranças.

Palavras-chave: Atenção Primária à Saúde; Planejamento estratégico; Gestão em saúde.

\begin{abstract}
Objective: To evaluate how the Situational Strategic Planning in Primary Health Care (PHC) occurs in Brazil, and how it contributes to health management. Method: This is an integrative literature review carried out from November 2020 to April 2021. The inclusion criteria of the studies were: articles in Portuguese, English, and Spanish, published in the last 05 years, that presented considerations about the importance of situational strategic planning in the management within PHC, indexed in the LILACS and PUBMED databases, using the subject descriptors "primary health care" and "strategic planning". Publications in the format of thesis, dissertation, book chapter and those unrelated to the research topic were excluded. Results: 170 publications were found. After applying the inclusion and exclusion criteria, 9 articles were obtained from which themes emerged regarding the importance of Situational Strategic Planning and how it should be articulated in various areas within PHC management, promoting goals and indicators capable of evaluating and monitoring results in planning. Conclusion: The results of this review found different ways of applying the Situational Strategic Planning (SSP) in the management of primary health care with a focus on promotion and prevention, which showed that there are still many challenges to be faced, however, possible to be addressed through the integration of teaching and service, the co-responsibility of the participants and the improvement of this management skills in professionals who work in the leaderships.
\end{abstract}

Keywords: Primary Health Care; Strategic planning; Health management.

\title{
Resumen
}

Objetivo: Evaluar cómo ocurre la Planificación Estratégica Situacional en la Atención Primaria de Salud (APS) en Brasil y cómo contribuye a la gestión en salud. Método: Se trata de una revisión integrativa de la literatura realizada de noviembre de 2020 a abril de 2021. Los criterios de inclusión de los estudios fueron: artículos en portugués, inglés y español, publicados en los últimos 05 años, que presentaron en su discusión consideraciones sobre la importancia de la planificación estratégica situacional en la gestión dentro de la APS, indexada en las bases de datos LILACS y PUBMED, utilizando como descriptores temáticos "atención primaria de salud" y "planificación estratégica". Se excluyeron las publicaciones en formato de tesis, disertación, capítulo de libro y aquellas que no tuvieran relación con el tema de investigación. Resultados: se encontraron 170 publicaciones. Después de aplicar los criterios de inclusión y exclusión, se obtuvieron 09 artículos de los cuales surgieron temas sobre la importancia de la Planificación Estratégica Situacional y cómo debe articularse en las diversas áreas dentro de la gestión de la APS, promoviendo metas e indicadores capaces de evaluar y monitorear los resultados en la planificación. Conclusión: Los resultados de esta revisión encontraron diferentes formas de aplicar la Planificación Estratégica Situacional (PES) en la gestión de la atención primaria de salud con enfoque de promoción y prevención, lo que mostró que aún hay muchos desafíos por enfrentar, sin embargo, posibles de ser trabajado a través de la integración de la docencia y el servicio, la corresponsabilidad de los participantes y la mejora de esta habilidad de gestión en los profesionales que actúan en el liderazgo.

Palabras clave: Atención Primaria de Salud; Planificación estratégica; Manejo de la salud.

\section{Introdução}

Um novo modelo de atenção à saúde construído no Brasil a partir de 1986 trouxe à necessidade de uma reforma sanitária que oferecesse uma estratégia de reorganização substitutiva ao modelo tradicional fragmentado executado empiricamente até então. Para tanto, segundo Campos (2012), as diretrizes de construção desse novo modelo de atenção à saúde, baseado nos princípios de universalidade, integralidade, equidade e descentralização seriam ratificadas mediante a elaboração na Constituição Federal de 1988 através da criação do Sistema Único de Saúde (SUS).

Neste novo modelo de atenção à saúde emergiu uma "rede integrada regionalizada e hierarquizada de serviços, constituindo um sistema único, organizado, descentralizado, com direção única em cada esfera de governo, prestando atendimento integral, a partir da priorização de atividades preventivas, sem prejuízo das assistenciais e com participação popular" (Conil, 2004).

Foi aprovada a Política Nacional de Atenção Básica (PNAB) pela Portaria n 2.488 de 21 de outubro de2011, com objetivo de reorganizar o sistema de saúde a partir da atenção primária numa rede de atenção à saúde de modo horizontal. 
Conforme Bursztyn et al. (2010) a reorientação dos setores de saúde utilizando a APS como componente fundamental cria um sistema conduzido por ações sanitárias mais eficazes e eficientes, objetivando fazer cumprir o que é preconizado pela lei.

Neste novo formato de atenção à saúde, um novo modelo de gestão foi implantado, buscando maior participação de todos os integrantes do sistema. Portanto, usa-se a proposta da realização do Planejamento Estratégico Situacional (PES) para garantir que os problemas da Atenção Primária à Saúde fossem alcançados com melhorias. O planejamento estratégico envolve uma análise do ambiente externo, uma análise do ambiente interno e o desenvolvimento ou refinamento das estratégias direcionais organizacionais (Artmann, 2000).

Entre as vertentes do planejamento estratégico que surgem na América Latina a partir dos anos 70, destaca-se o PES, de Carlos Matus, autor chileno, que a partir de sua vivência como ministro da Economia do governo Allende, no período de 1970-73, e da análise de outras experiências de planejamento normativo ou tradicional cujos fracassos e limites instigaram um profundo questionamento sobre os enfoques e métodos utilizados. Porém, segundo Artmann (2000), este método vem sendo adotado em outras áreas como a saúde, educação e planejamento urbano, por exemplo.

Partindo da complexidade, da fragmentação e da incerteza que caracterizam os processos sociais, que se assemelham a um sistema de final aberto e probabilístico, onde os problemas se apresentam, em sua maioria, não estruturados e o poder se encontra compartido, ou seja, nenhum ator detém o controle total das variáveis que estão envolvidas na situação (Artmann, 2000).

Considerando a importância da APS para a reorganização dos serviços de saúde do país e seu papel ante a promoção e a prevenção de muitas doenças, buscou-se, neste artigo, responder a seguinte questão de pesquisa: Quais são as evidências científicas sobre PES e sua importância como ferramenta de prevenção e promoção na gestão da Atenção Primária a Saúde?

Realizou-se uma revisão integrativa da literatura (RIL), que teve por objetivo verificar e descrever publicações acerca da importância do PES como ferramenta para obter sucesso na prevenção a partir da gestão na APS.

Neste contexto, os métodos meta-científicos são apontados como uma maneira eficaz para avaliação do conjunto da produção científica em uma determinada área do conhecimento. Tal tipo de avaliação é feita a partir da revisão de informações em fontes de publicação científica, bases de indexação de dados, bancos de teses e dissertações, periódicos científicos, gerando indicadores importantes da produção, sintetizando as principais ideias, hipóteses, métodos, resultados, bem como o impacto da produção. Nesse sentido, a revisão integrativa permite a busca, a avaliação crítica e a síntese das evidências disponíveis do tema investigado (Ganong, 1987; Whittemore \& Knaf, 2005).

\section{Metodologia}

Tratou-se de uma pesquisa qualitativa, sob o método de revisão integrativa da literatura, realizada no período de novembro de 2020 a abril de 2021. Esta possibilita a síntese de diversos estudos e permite a obtenção de conclusões gerais a respeito de um determinado assunto. Ela compreende seis etapas: identificação do tema e questão de pesquisa para a revisão; estabelecimento de critérios de inclusão e exclusão de estudos; categorização; avaliação; interpretação dos resultados dos estudos e a apresentação da revisão (Mendes et al., 2008). Os critérios de inclusão dos estudos foram: artigos em português, inglês e espanhol, publicados nos últimos 5 anos, que apresentassem em sua discussão considerações sobre a importância do planejamento estratégico na gestão dentro da APS, indexados nas bases de dados LILACS e PUBMED, utilizando-se como descritores de assunto "primary healthcare" e "planification stratégique". Foram excluídas as publicações no formato de tese, dissertação, capítulo de livro e as que não tinham relação com o tema da pesquisa.

Visando à sistematização dos dados, desenvolveu-se um instrumento de coleta contendo: autores, ano, local, título do artigo, características metodológicas dos artigos e resultados alcançados. Após coleta dos dados, procedeu-se à caracterização do corpus de análise. Conforme apresentado no fluxograma 1. 
Fluxograma 1: Processo de produção da Revisão Integrativa de Literatura sobre Planejamento Estratégico Situacional de publicações de 2015 a 2020, Belém - Pará.

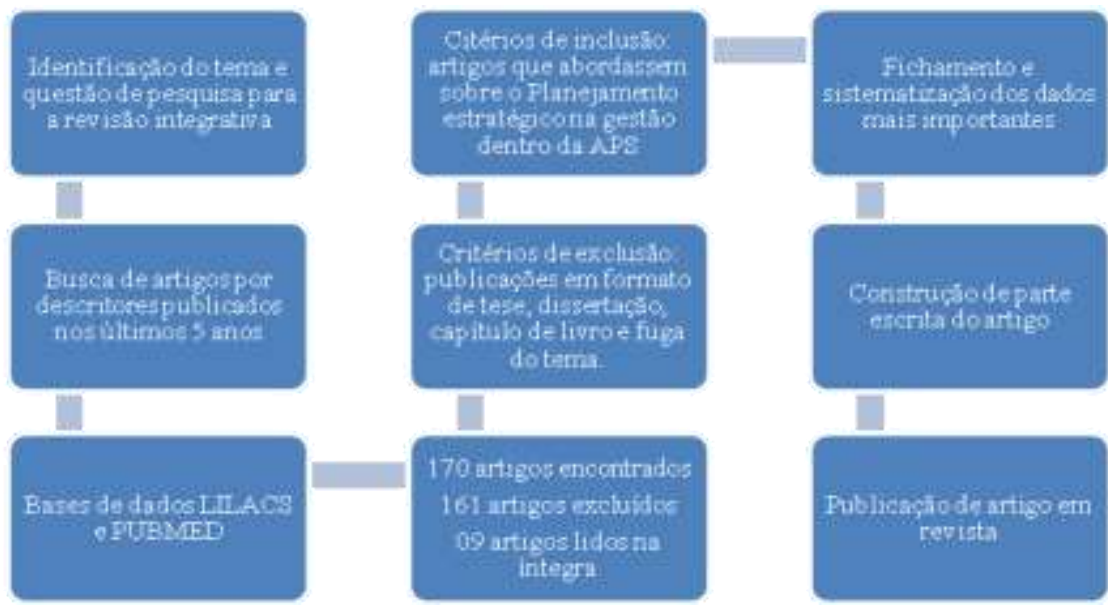

Fonte: Autores (2021).

\section{Resultados}

Foram encontrados 170 artigos na busca inicial, entretanto, somente 09 estavam de acordo com os critérios de elegibilidade do presente estudo.

As informações com relação aos autores, periódicos, ano de publicação, métodos e resultados dos estudos encontrados foram agrupados e apresentados nos quadros 1 e 2 a seguir. Quanto às conclusões das produções analisadas, foram enfatizadas a importância do PES e como ele deve ser articulado em diversas áreas dentro da gestão na Atenção Primária a Saúde promovendo sucesso nos objetivos traçados no planejamento.

Quadro 1 - Artigos selecionados sobre Planejamento Estratégico publicados no período de 2015 a 2020, Belém - Pará.

\begin{tabular}{|c|c|c|c|c|}
\hline $\mathbf{N}^{\circ}$ & Título & Autores & Ano & Periódico \\
\hline 1 & $\begin{array}{l}\text { Relato de experiência: utilizando o Planejamento } \\
\text { Estratégico Situacional (PES) como ferramenta de } \\
\text { gestão }\end{array}$ & Andrigue et al. & 2016 & $\begin{array}{l}\text { Brazilian Journal of Surgery } \\
\text { and Clinical Research - } \\
\text { BJSCR }\end{array}$ \\
\hline 2 & $\begin{array}{l}\text { Planejamento Estratégico como exigência ética para a } \\
\text { equipe e a gestão local da atenção básica em saúde. }\end{array}$ & Junges et al. & 2015 & $\begin{array}{l}\text { Interface- comunicação, } \\
\text { saúde, educação }\end{array}$ \\
\hline 3 & $\begin{array}{l}\text { Liderazgo y gestión estratégica en sistemas de salud } \\
\text { basados en atención primária de salud }\end{array}$ & Puertas et al. & 2020 & Rev panam salud publica \\
\hline 4 & $\begin{array}{l}\text { Planejamento estratégico e implantação dos testes } \\
\text { rápidos de HIV, sífilis e hepatites virais em uma } \\
\text { capital brasileira: relato de experiência. }\end{array}$ & Teixeira et al. & 2015 & Rev Bras em prom da saúde \\
\hline 5 & $\begin{array}{l}\text { Planejamento estratégico na promoção à saúde do } \\
\text { idoso: Uma experiência integradora academia- } \\
\text { serviço- comunidade }\end{array}$ & Santos et al. & 2019 & Rev. Inter educ saúde \\
\hline 6 & $\begin{array}{l}\text { Construindo cidades saudáveis: A instrumentalização } \\
\text { de políticas públicas intersetoriais de saúde a partir } \\
\text { do planejamento estratégico situacional }\end{array}$ & Lima et al. & 2020 & Saúde e sociedade \\
\hline 7 & $\begin{array}{l}\text { Vias do planejamento na transferência do tratamento } \\
\text { diretamente observado da tuberculose }\end{array}$ & Carvalho et al & 2018 & $\begin{array}{ll}\text { Rev Latino- am. } \\
\text { Enfermagem }\end{array}$ \\
\hline 8 & $\begin{array}{l}\text { Gerando conversas significativas: World Café no } \\
\text { planejamento estratégico interprofissional em } \\
\text { educação permanente }\end{array}$ & Bazilio et al. & 2020 & Rev. Bras. Enferm. \\
\hline 9 & $\begin{array}{l}\text { Os desafios do planejamento municipal a partir da } \\
\text { perspectiva de enfermeiras gestoras }\end{array}$ & Reuter et al. & 2020 & Rev. Bras. Enferm \\
\hline
\end{tabular}

Fonte: Autores (2021). 
Quadro 2 - Objetivo, Método e Resultados dos artigos selecionados para revisão integrativa de literatura sobre Planejamento Estratégico na Atenção Primária à Saúde, de janeiro 2020 a abril de 2021, Belém- Pará.

\begin{tabular}{|c|c|c|c|}
\hline Autores & Objetivo & Métodos & Resultados \\
\hline Andrigue et al. & $\begin{array}{l}\text { Descrever um processo de planejamento } \\
\text { participativo, realizado no mês de junho de } \\
2018 \text {, por residentes do Programa de } \\
\text { Residência Multiprofissional em Saúde da } \\
\text { Família e Comunidade, em uma Unidade } \\
\text { Básica de Saúde. }\end{array}$ & $\begin{array}{l}\text { Trata-se de um relato de experiência } \\
\text { descritivo e reflexivo sobre os aspectos } \\
\text { vivenciados durante a construção de um } \\
\text { plano de ações utilizando o Planejamento } \\
\text { Estratégico Situacional, conforme elaborado } \\
\text { por Carlos Matus. }\end{array}$ & $\begin{array}{l}\text { Demonstrou na prática que a integração } \\
\text { ensino-serviço na construção de um PES } \\
\text { serviu para melhorar a qualidade nos } \\
\text { atendimentos em uma unidade de saúde. }\end{array}$ \\
\hline Junges et al. & $\begin{array}{l}\text { Discutir no âmbito da bioética, a } \\
\text { necessidade de atenção básica } \\
\text { complementar a deliberação moral da } \\
\text { clínica com a exigência ética do } \\
\text { planejamento estratégico da gestão local, } \\
\text { articulado com as ações da vigilância em } \\
\text { saúde e a macrogestão do sistema de } \\
\text { saúde. }\end{array}$ & $\begin{array}{l}\text { Ocorre a apresentação de um caso concreto } \\
\text { complexo de atenção básica onde estão } \\
\text { implicadas as etapas propostas por Matus, } \\
\text { sobre o Planejamento estratégico: cognitivo; } \\
\text { valorativo; operativo e avaliativo. }\end{array}$ & $\begin{array}{l}\text { Defende a tese de que a efetividade da } \\
\text { deliberação moral da clínica, no âmbito } \\
\text { da atenção básica, precisa incorporar, em } \\
\text { seus cursos de ação, os processos de } \\
\text { planejamento estratégico em interação } \\
\text { com a macrogestão e com a vigilância em } \\
\text { saúde. }\end{array}$ \\
\hline Puertas et al. & $\begin{array}{l}\text { Identificar e analisar vários tipos de } \\
\text { liderança e gestão que pode contribuir para } \\
\text { o fortalecimento dos sistemas de saúde } \\
\text { baseados em atenção primária em saúde } \\
\text { (APS). }\end{array}$ & $\begin{array}{l}\text { Foi realizada uma revisão estruturada da } \\
\text { literatura sobre gestão e liderança aplicável } \\
\text { aos sistemas de saúde baseados na APS. }\end{array}$ & $\begin{array}{l}\text { Líderes com inteligência emocional } \\
\text { devem ser estrategistas para liderar o } \\
\text { sistema em sua transição para } \\
\text { organizações resilientes, e "alquimistas". }\end{array}$ \\
\hline Teixeira et al. & $\begin{array}{l}\text { Descrever a experiência da implementação } \\
\text { do planejamento estratégico para o } \\
\text { processo de promoção dos testes rápidos } \\
\text { de HIV, sífilis e hepatites B e C em uma } \\
\text { capital brasileira. }\end{array}$ & $\begin{array}{l}\text { Buscou-se criar um plano operativo, a fim de } \\
\text { elaborar estrategicamente o trabalho de uma } \\
\text { equipe, com o intuito de promover a } \\
\text { testagem rápida na rede SUS. }\end{array}$ & $\begin{array}{l}\text { Mostrou o desenvolvimento de um plano } \\
\text { operativo fundamentado no PES, } \\
\text { revelando a sua aplicabilidade como } \\
\text { ferramenta de gestão e indispensável ao } \\
\text { trabalho em equipe. }\end{array}$ \\
\hline Santos et al. & $\begin{array}{l}\text { Discutir o envolvimento de diversos atores } \\
\text { sociais na construção coletiva do } \\
\text { Planejamento Estratégico Situacional em } \\
\text { um componente curricular dos cursos de } \\
\text { graduação em saúde e destacar os } \\
\text { potenciais benefícios desse processo. }\end{array}$ & $\begin{array}{l}\text { Construção do diagnóstico situacional de um } \\
\text { grupo de idosos da USF e realização de um } \\
\text { Planejamento Estratégico Situacional (PES), } \\
\text { segundo Carlos Matus, de intervenções } \\
\text { comunitárias com base na Educação Popular } \\
\text { em Saúde. }\end{array}$ & $\begin{array}{l}\text { A experiência de PES em Saúde, em } \\
\text { consonância a Educação Popular, } \\
\text { proporcionou um olhar crítico frente às } \\
\text { necessidades de saúde, pois, nessa } \\
\text { pesquisa, estudantes puderam } \\
\text { desenvolver a escuta qualificada, } \\
\text { elaboraram todo o percurso com base nas } \\
\text { etapas do PES. }\end{array}$ \\
\hline Lima et al. & $\begin{array}{l}\text { Apresentar o Planejamento Estratégico } \\
\text { Situacional como uma metodologia para } \\
\text { construção de cidades saudáveis. }\end{array}$ & Relato de experiência. & $\begin{array}{l}\text { O PES pode contribuir para o } \\
\text { fortalecimento das políticas públicas de } \\
\text { saúde, e para a construção de cidades } \\
\text { saudáveis pela instrumentalização de um } \\
\text { processo de planejamento democrático e } \\
\text { inclusivo. }\end{array}$ \\
\hline Carvalho et al. & $\begin{array}{l}\text { Investigar o processo de planejamento da } \\
\text { transferência da política do Tratamento } \\
\text { Diretamente Observado da tuberculose. }\end{array}$ & $\begin{array}{l}\text { Estudo qualitativo, realizado por meio de } \\
\text { entrevistas e roteiro semi-estruturado } \\
\text { aplicado a cinco sujeitos, dentre } \\
\text { coordenadores e gestores dos programas de } \\
\text { controle da tuberculose e o secretário de } \\
\text { saúde de um município do Sul do Brasil. O } \\
\text { Planejamento Estratégico Situacional e a } \\
\text { Análise de Discurso de matriz francesa } \\
\text { foram os referenciais teórico e analítico } \\
\text { utilizados, respectivamente. }\end{array}$ & $\begin{array}{l}\text { O PES como alicerce operacional } \\
\text { fundante para o processo de transferência } \\
\text { de políticas públicas visando o controle } \\
\text { adequado. }\end{array}$ \\
\hline Bazilio et al. & $\begin{array}{l}\text { Apresentar as particularidades do World } \\
\text { Café identificando os principais aspectos } \\
\text { que caracterizam uma estratégia de } \\
\text { planejamento para um diálogo construtivo, } \\
\text { interativo e participativo dos sujeitos, com } \\
\text { vistas ao aprendizado colaborativo e } \\
\text { inovador. }\end{array}$ & $\begin{array}{l}\text { Realizou-se uma oficina de trabalho com o } \\
\text { método World Café, com } 24 \text { profissionais } \\
\text { das equipes que atuam em unidades básicas } \\
\text { de saúde. }\end{array}$ & $\begin{array}{l}\text { Mostrou a aplicação do método WC na } \\
\text { prática do PES atendendo às necessidades } \\
\text { de construção coletiva e promoveu troca } \\
\text { de conhecimento entre os participantes, } \\
\text { favorecendo o trabalho colaborativo }\end{array}$ \\
\hline Reuter et al. & $\begin{array}{l}\text { Analisar o planejamento municipal } \\
\text { desenvolvido } \\
\text { coordenadoras da APS, voltado para o } \\
\text { enfrentamento das Doenças Crônicas Não } \\
\text { Transmissíveis, sob a perspectiva do PES. }\end{array}$ & $\begin{array}{l}\text { Estudo exploratório descritivo, com } \\
\text { abordagem qualitativa, desenvolvido em } \\
\text { municípios de uma Região de Saúde do Rio } \\
\text { Grande do Sul. A análise de conteúdo foi a } \\
\text { temática e a perspectiva teórica de análise } \\
\text { estratégica. }\end{array}$ & $\begin{array}{l}\text { Indica que é necessário um projeto } \\
\text { permanente de PES na atenção à saúde } \\
\text { aos doentes crônicos e que as enfermeiras } \\
\text { que assumam cargos de gestão em nível } \\
\text { municipal já possuam apropriação teórica } \\
\text { e conceitual em relação a esta prática }\end{array}$ \\
\hline
\end{tabular}

Fonte: Autores (2021).

\section{Discussão}

No estudo de Andrigue et al. (2016), realizada pelo curso de enfermagem da Universidade Comunitária da Região Chapecó (UNOCHAPECO), buscou descrever a utilização do PES como ferramenta de gestão capaz de aproximar estudantes 
da realidade diária das equipes de saúde possibilitando, através da participação ativa, a construção do conhecimento. Ao término deste estudo observou-se que o PES é capaz de qualificar a prática assistencial melhorando o atendimento ao usuário e que a verdadeira integração ensino serviço é capaz de transformar as práticas de trabalho e de formação de forma contínua e conjunta no sentido de superar o modelo de saúde centrado na doença e em práticas, predominantemente, reativas, com a finalidade de reorganizar o acesso.

Tratando-se de integração entre a clínica e a vigilância na atenção básica e a consequente necessidade da aproximação entre atenção e gestão a qual demonstra dificuldades de execução devido cada uma responder a lógicas diversas, o estudo de Junges et al. (2015), trouxe à tona que apesar da diversidade dos problemas encontrados na saúde coletiva, o planejamento estratégico abre caminhos virtuosos para a efetividade da deliberação clínica no contexto da atenção básica através da corresponsabilização das ações incorporando a interação da macrogestão com a vigilância em saúde.

No estudo sistemático de Puertas et al. (2020) observou-se que o planejamento estratégico e a gestão facilitam o desenvolvimento de uma identidade comum, embora algumas organizações sejam super gerenciadas e mal lideradas, à saúde universal funciona como um impulsionador estratégico que requer liderança transformacional no sentido de avançar em direção aos sistemas baseados na APS. A liderança nesses sistemas deve focar na identificação de desafios adaptativos, articulando as equipes e protegendo as lideranças de base, além de se adaptar aos tempos e aliar a lógica de ação do estrategista para a transformação do setor. Os modelos de qualidade estabelecem critérios de liderança semelhantes a outras teorias, enfatizando a inovação e a identidade organizacional.

Em um trabalho em equipe, o PES funciona como uma ferramenta de gestão indispensável para que haja êxito nos objetivos propostos. Foi o que o estudo de Teixeira, Nascimento et al. (2016) realizado no município de Belo Horizonte-MG concluiu que o desenvolvimento do Plano Operativo fundamentado no Planejamento Estratégico Situacional demonstrou que ele pode ser utilizado para a melhoria da promoção de saúde por meio da efetividade dos testes rápidos aumentando a cobertura do programa de testagem rápida no município.

No que tange ao cuidado em saúde, a experiência de PES, em consonância com os princípios da Educação Popular, proporcionou a estudantes de uma pesquisa (Santos et al., 2019) um olhar crítico frente às necessidades de saúde apresentadas por idosos, visto que os pesquisadores puderam desenvolver a escuta qualificada, além de compreender e elaborar todo o percurso com base nos momentos do PES com a participação de todos os atores, possibilitando a horizontalidade das relações em todas as etapas, desde o planejamento até a execução das atividades.

O PES também pode contribuir para o fortalecimento das políticas públicas de saúde, e para a construção de cidades saudáveis pela instrumentalização de um processo de planejamento democrático e inclusivo. Em seu estudo, Lima (2020) objetivou apresentar o PES como uma metodologia para construção de cidades saudáveis. Desde a definição do problema, a apreciação situacional, elaboração do plano até a avaliação. Ele afirma que o desafio é construir cidades saudáveis para todos e não para alguns, cidades mais justas e menos iníquas, territórios de inclusão e não de segregação. Mas também retrata que o setor de saúde sozinho não é capaz de construir a cidade saudável, a não ser na intersetorialidade, o que demanda integração de políticas e planejamento.

É notória a importância do Planejamento Estratégico como ferramenta de gestão na promoção em saúde. Entretanto, essa prática deve ser trabalhada com certo conhecimento para que não haja prejuízo nos resultados esperados. No estudo desenvolvido na cidade de Porto Alegre-RS, Peruhype et al. (2018) cujo objetivo principal foi de investigar o planejamento da transferência do Tratamento Domiciliar Observado (TDO) na capital gaúcha, os entrevistados apresentaram certo despreparo quando questionados quanto à forma de planejamento. Por tratar-se ainda de grave problema de saúde pública, a tuberculose exige um olhar mais cauteloso para o processo de transferência de políticas que visem o seu controle, sendo o planejamento dessa etapa um alicerce operacional fundante. Assim, a notada assistematização do planejamento para a execução da 
transferência do TDO indica não apenas a fragilidade dessa atividade, mas também a possibilidade de sua inexistência. Não raro são observadas ações planejadas que nem sempre são executadas e exigências rotineiras do "apagar incêndios", que não apenas desvirtuam e inviabilizam a prática do planejamento, mas, sobretudo, reforçam uma prática gestora pouco reflexiva e fundamentada (Peruhype, 2018).

A importância do planejamento no controle da TB é comprovada cientificamente. No Brasil, o próprio Programa Nacional de Controle da Tuberculose busca aprimorar a qualificação de processos como o de planejamento, monitoramento, avaliação das ações de controle, de prevenção, assistência, diagnóstico, vigilância epidemiológica etc.

Em busca de inovações que envolvem o planejamento estratégico, o estudo de Bazilio et al. (2020) cujo objetivo foi apresentar as particularidades do World Café identificando os principais aspectos que caracterizam uma estratégia de planejamento para um diálogo construtivo, interativo e participativo dos sujeitos, com vistas ao aprendizado colaborativo e inovador, demonstrou-se muito eficaz na construção de um planejamento das ações de educação permanente conjunto entre diferentes unidades básicas de saúde e interprofissionais. Atendeu às necessidades de construção coletiva e, de forma efetiva, promoveu troca de conhecimento entre os participantes, favorecendo o trabalho colaborativo. Tal iniciativa aumentou a adesão dos profissionais de saúde aos processos de formação profissional no âmbito do trabalho, pois eles participaram desde o planejamento até a execução das ações em educação permanente. Além disso, ela foi constituída pela real necessidade identificada, vivenciada e organizada por esses profissionais, com o apoio deste método.

$\mathrm{Na}$ condição de gestor ou equipe gestora, um dos grandes desafios é desenvolver coletivamente um planejamento que contribua para melhorar a saúde da população de seu território, agregar adesão das equipes, atingirem resultados e, assim, fortalecer o SUS. Processos coletivos são complexos e precisam ser estrategicamente trabalhados (Gil, Luiz, Gil; 2016).

Planejar, portanto, passa a ser uma necessidade cotidiana, um processo permanente para que se possam garantir diretrizes às ações desenvolvidas, corrigindo itinerários, enfrentando imprevistos e buscando sempre caminhar rumo aos objetivos desejados (Lacerda et al., 2016).

Segundo estes autores, praticamente todas as atividades da vida moderna que são vinculadas a uma ou mais organizações, necessitam de planejamento para obterem resultados em seus propósitos e objetivos. E a saúde que é um setor que articula diferentes unidades, programas e serviços não se difere, pois para garantir o cuidado à saúde de toda sociedade é necessário um grande esforço e muita competência de gestão que é possível somente através do planejamento.

O estudo de Matus (1996) realizou uma análise estratégica sobre a política, planejamento \& governo o qual retratou a importância de se conhecer o cenário para se transformar a realidade, a partir da leitura dos diferentes atores sociais, buscando a situação-objetivo de qualificar o planejamento (municipal, regional e estadual), no presente estudo, partindo da realidade local, no que tange ao adoecimento crônico.

Já Reuter et al. (2020), analisaram o planejamento municipal desenvolvido por enfermeiras coordenadoras da APS, voltado para o enfrentamento das doenças crônicas não Transmissíveis, sob a perspectiva do PES apresentou em seu resultado que ainda é necessário um projeto permanente de planejamento da atenção à saúde aos adoecidos crônicos e que as enfermeiras que assumam cargos de gestão em nível municipal já concentrem relevante apropriação teórica e conceitual em relação a esta prática a qual têm muito a contribuir com este processo no âmbito do SUS. Desta forma podemos finalizar reforçando que o planejamento em saúde pode ser compreendido como um dispositivo para sistematização das ações e racionalização dos processos de trabalho, visto que estes, a depender da realidade, são complexos e demandam organização prévia. Por este motivo, percebe-se que em um território, para se obter êxito e bons resultados, é necessário que os trabalhadores de saúde, usem suas habilidades e competências no reconhecimento das dinâmicas sociais e tenham capacidade de articulação entre os diferentes sujeitos envolvidos no arranjo do espaço (Gil et al., 2016). 
Em todos os estudos o PES aponta diferenças, revela os conflitos que podem estar ocultos que num primeiro encontro a situação pode parecer pior do que antes, levando em consideração que os membros podem ter aprendido a viver com a situação problema (Perera \& Peiró, 2012).

\section{Conclusão}

Os achados nesta revisão trouxeram à tona o que a literatura já condiz em relação aos benefícios do planejamento estratégico no que tange as ações voltadas à promoção e prevenção em saúde na atenção primária.

No contexto da aplicabilidade do planejamento estratégico no SUS, percebe-se com esta revisão integrativa, que ainda existem muitos desafios a serem enfrentados, porém, possíveis de serem trabalhados através da conexão ensino e serviço, da corresponsabilidade dos participantes e da aprimoração desta habilidade de gestão em profissionais que atuam em posições de liderança.

Ainda que seja um assunto de relevância e pertinência para a área de gestão, se fazem necessários mais estudos com abordagem focada no conhecimento e percepção sobre a utilização desta ferramenta na área da saúde tendo em vista que o planejamento em saúde é considerado um dispositivo para sistematizar ações que facilitam os processos de trabalho.

\section{Referências}

Andrigue, K. C. K., Hilleshein, A. C., Jochims, K. J. \& Marafon, S. P. (2016). Relato de Experiência: Utilizando o Planejamento Estratégico Situacional (PES) como Ferramenta de Gestão. Brazilian Journal of Surgery and Clinical Reseach BJSR. 15(4), 160-1. https://www.mastereditora.com.br/periodico/20191110_131936.pdf.

Arantes, P. \& Luiz, F. (2016). Planejamento estratégico e gestão por resultados: o caso do Ministério da Saúde. Physis - Revista de Saúde Coletiva, 26 (3),9811007. ISSN: 0103-7331. https://www.redalyc.org/articulo.oa?id=400848354014.

Artmann, E. (2000). O planejamento estratégico situacional no nível local: um instrumento a favor da visão multissetorial. Cadernos da Oficina Social 3: Série Desenvolvimento Local. Rio de Janeiro: Coppe/UFRJ.

Bazilio, J., Pereira, J. de A., Figueira, M. C. S. \& Silva, E. M. (2020). Gerando conversas significativas: World Café no planejamento estratégico interprofissional em Educação Permanente. Rev. Bras. Enferm. 73 (5): https://doi.org/10.1590/0034-7167-2019-0279

Bursztyn I., Kushnir, R., Giovanella, L., Stolkiner, A., Sterman-Heimann, L. \& Riveros, M. I. (2010) Notas para el estudio de la atención primaria en contextos de sistemas de salud segmentados. RevSalud Pública]; 12(1):77-88. https://www.arca.fiocruz.br/handle/icict/44994

Campos, A. (2012). Planejamento estratégico situacional na atenção básica: solucionando problemas e reinventando ações -. Revista Brasileira em Promoção da Saúde. 22. 10.5020/554. https://www.researchgate.net/publication/307765922_Planejamento_estrategico_situacional_na_atencao_basica_solucio nando_problemas_e_reinventando_acoes_-_doi105020180612302009p151

Conill, E. M. (2004). Avaliação da integralidade: conferindo sentido para os pactos na programação de metas dos sistemas municipais de saúde. Cad Saúde Pública. 2004; 20 (5):1417-23.

Figueiredo, I. D. T., Torres, G. M.C., Cândido, J. A. B., Morais, A. P. P., Pinto, A. G. A. \& Almeida, M. I. (2020). Planejamento estratégico como ferramenta de gestão local na atenção primária à saúde. Revista Família, Ciclos de Vida e Saúde no Contexto Social, 8(1),27-38. https://www.redalyc.org/articulo.oa?id=497962779006

Ganong, L. H. (1987). Integrative reviews of nursing research. Research in Nursing \& Health, 10(1), 11.

Gil, C. R. R., Luiz, I. C. \& Gil, M. C. R. (2016). Gestão pública em saúde: a Importância do planejamento na gestão do SUS. EDUFMA: Maranhão.

Junges, J. R., Barbiani \& Zoboli, E. L. C. P. (2015). Planejamento Estratégico como exigência ética para a equipe e a gestão local da Atenção Básica em Saúde. Interface. 19(53), 265-274. https://doi.org/10.1590/1807-57622014.0331.

Lacerda, J.T., Botelho, L.J., Colussi, C.F. (2016). Planejamento na atenção básica [Recurso eletrônico] Especialização Multiprofissional na Atenção Básica Modalidade a Distância. / Universidade Federal de Santa Catarina. - Florianópolis, Universidade Federal de Santa Catarina.

Lima, F. \& Antunes, L. S. C. (2020). Construindo cidades saudáveis: a instrumentalização de políticas públicas intersetoriais de saúde a partir do Planejamento Estratégico Situacional. Saude soc; 29 (2): e 200058, https://doi.org/10.1590/S0104-12902020200058

Matus, C. (1996). Política, planejamento \& governo. Ipea.

Mendes, C. D. S., Silveira, R.C.C.P., \& Galvão, C.M. (2008). Revisão integrativa: método de pesquisa para a incorporação de evidências na saúde e na enfermagem. Tex. Cont. Enf. 17(4): https://doi.org/10.1590/S0104-07072008000400018 
Research, Society and Development, v. 11, n. 2, e5911225302, 2022

(CC BY 4.0) | ISSN 2525-3409 | DOI: http://dx.doi.org/10.33448/rsd-v11i2.25302

Peruhype, R. C., Mitano F., Hoffmann, J. F., Surniche, C. A. \& Palha, P. F. (2018). Vias do planejamento na transferência do tratamento diretamente observado da tuberculose. Rev. Latino-Am. Enfermagem. 26 e 3015. https://doi.org/10.1590/1518-8345.2213.3015.

Perera, F. P. F.\& Peiró, M. (2012). Strategic Planning in Healthcare Organizations. Rev Esp Cardiol (Engl Ed) 65(8):749-54.

Puertas, E. B., \& Sotelo, J. M., (2020). Ramos G. Liderança e gestão estratégica em sistemas de saúde baseados na atenção primária à saúde. Rev Panam Salud Publica; 44 e124. 10.26633 / RPSP.2020.124

Reuter, C. L. O., Maciel P. P., Santos V. C. F., Riquinho D. L., \& Ramos A. R. (2020). Os desafios do planejamento municipal a partir da perspectiva de enfermeiras gestoras. Rev. Bras. Enferm. 73(2): https://doi.org/10.1590/0034-7167-2018-0409.

Santos, C. M. L., Ferreira S. M. S. P., Almeida C. S., Araújo I. B., Marino A. C., \& Menezes V. M. (2019). Planejamento estratégico na promoção à saúde do idoso: uma experiência integradora academia-serviço-comunidade. Rev Inter Educ Saúde. 3(1):36-44. http://dx.doi.org/10.17267/2594-7907ijhe.v3i1.2139.

Moraes, T. J., \& Nascimento, R. L. F. (2016). Planejamento estratégico e implantação dos testes rápidos de hiv, sífilis e hepatites virais em uma capital brasileira: relato de experiência. Revista Brasileira em Promoção da Saúde, 29(1),139-144. 1806-1222. https://doi.org/10.5020/18061230.2016.p139.

Whittemore., \& Knaf, K. (2005). The integrative review: updated methodology Robin. Methodological Issues In Nursing Research, 52(5), 546-553. https://doi.org/10.1016/j.pmn.2007.11.006. 